\title{
Universality in the Electroproduction of Vector Mesons
}

\author{
V.L. Baltar \\ Departamento de Física, Pontifícia Universidade Católica do Rio de Janeiro \\ Rio de Janeiro 22453-900, RJ, Brazil
}

H.G. Dosch

Institut für Theoretische Physik, Philosophenweg 16, D-6900 Heidelberg, Germany

E. Ferreira

Instituto de Física, Universidade Federal do Rio de Janeiro C.P. 68528, Rio de Janeiro 21941-972, RJ, Brazil

\begin{abstract}
We study universality in the electroproduction of vector mesons using a unified nonperturbative approach which has already proved to reproduce extremely well the available experimental data. In this framework, after the extraction of factors that are specific of each vector meson, we arrive at a reduced integrated elastic cross section which is universal. Our calculations suggest a finite infrared behavior for the strong coupling constant.
\end{abstract}




\section{INTRODUCTION}

Experiments show a striking similarity among the production cross sections of the various vector mesons, pointing to the existence of a universal content in these processes. In the present work we study this universality property using a nonperturbative procedure which describes in a unified way the cross sections for elastic electroproduction of the S-wave vector mesons.

We employ a general treatment of high energy scattering based on the functional integral approach to QCD [1, 2, 3] and on the WKB method. The functional integrals are calculated nonperturbatively, in an extended stochastic vacuum model [4, 5]. This nonperturbative approach has been successfully applied in many fields, from hadron spectroscopy to high energy scattering [6, 17, 8], DVCS [9] and structure functions [10]. Recently, the method has been shown to provide a good description of the existing observed data on vector meson electroproduction and a comparison with the characteristic features of the perturbative approaches [11, 12, 13, 14, 15, 16, 17, 18] has been extensively discussed [19].

In our scheme the structure of the transition amplitude is defined in terms of color neutral quark-antiquark states (color dipoles) that displace themselves in the external QCD vacuum field. The fundamental elementary dynamical effect is given by the loop-loop interaction: Wilson loops formed by dipoles in the photon-meson overlap product and in the proton (for simplicity treated in a diquark model). The transition to observable electroproduction amplitudes of hadrons is obtained through a superposition of the loop-loop amplitudes with the light cone wave functions of the hadrons and the photon used as weights. This procedure allows the calculation of polarized transition amplitudes and angular distributions within a unified view of all processes of vector meson electroproduction. We need no external inputs, and the only intervening quantities are inherently calculated in the nonperturbative approach; no new free parameters have to be introduced. A more detailed description of the method can be found in [6].

In order to look for the structure of the universal behavior that appears in the data we concentrate on the photon virtuality $\left(Q^{2}\right)$ dependence of the integrated elastic cross sections. This is the simplest observed quantity, determined almost fully by the shape of the photon and vector meson wave functions, both described as 
packets of quark-antiquark dipoles. Universality is inherent to the nonperturbative nature of the method here applied, and is reproduced accurately by our theoretical calculations.

We first consider the loop-loop amplitude which depends on the dipole sizes and orientation and on the impact parameter. The amplitude for the hadronic process in the impact parameter space is obtained by integrating the square of the loop-loop amplitude over the orientation and sizes of the dipoles, with the above mentioned weight factors, i.e., the squares of the proton wave function and the square of the overlaps of photon and meson wave functions. A Fourier transform leads from the impact parameter picture in the transverse plane to the momentum transfer description. After integration over the geometric weighted distribution of loops, the correct $Q^{2}$ dependence and normalization of cross sections emerge [20], which turns out to have form $\sigma=A_{V} /\left(1+Q^{2} / M_{V}^{2}\right)^{n}$, where $M_{V}$ is the vector meson mass. We notice that this essential variable arises naturaly in our calculations, in spite of $M_{V}$ not being introduced a priori in this framework. Actually the vector meson mass appears numerically only through the expression, given in the next section, which relates the electromagnetic coupling $f_{V}$ to the measured decay rate $\Gamma_{V \rightarrow e^{+} e^{-}}$.

The numerical factors $A_{V}$ that fix the normalization of the cross sections, as well as the power $n$, are determined uniquely by the model, without free parameters, reproducing the data with good precision. The values of $n$ differ little from one vector meson to another[20]. Predicted cross sections cover a range of 5 orders of magnitude [19].

The previously known property regarding universality is that, divided by the square of the quark charges of the vector mesons, (in the $\rho$ and $\omega$ meson cases, formed with differents quarks $\mathrm{u}$ and $\mathrm{d}$, this means the average effective charges), the values of the constans $A_{V}$ are quite similar.

We here show an important improvement of this universality property: instead of extracting the squared charges, we extract the squared electromagnetic couplings $f_{V}^{2}$. Besides leading to a closer grouping of the cross section values, this criterium to reach universality is more fundamental, as the couplings fix the properties of the wave functions. The electromaqnetic coupling, experimentally accessible through the electromagnetic decay rates, is directly related to the value of the wave function at the origin. The interesting comparison of the universality in cross section values 
obtained with extraction of squared charges and with extraction of squared couplings is shown in section 3 .

We then go a step further, and examine the expression that relates the couplings $f_{V}$ to the experimental electromagnetic decay rates $\Gamma_{V \rightarrow e^{+} e^{-}}$. A first order radiative correction to the decay rate [21, 22] introduces a factor

$$
\left[1-\left(\frac{16}{3 \pi}\right) \alpha_{S}\left(m_{f}\right)\right]
$$

with respect to the bare decay rate. The situation is that the fundamental universality property should be found with extraction of the pure coupling, $f_{V}^{(0)}$, not of the effective one $f_{V}$, with the radiative correction factor.

The first order radiative correction to the decay rate depends on the intervening quark mass, because of the running property of the strong coupling constant $\alpha_{S}\left(m_{f}\right)$. This quantity is known for values of the argument $m_{f}$ that are larger than about $1 \mathrm{GeV}$. Thus the bare coupling can be numerically determined for the charm and bottom cases. We show that, using the resulting values of bare couplings in $J / \psi$ and $\Upsilon$ decays, perfect matching of the corresponding reduced cross sections is obtained.

For the light mesons we have to consider effective values of the strong coupling constant. Studies about the infrared regularization of the gluon propagator point to finite values for $\alpha_{S}(0)$. We explore the possible criterion that the complete universality in the cross sections fixes effective values for $\alpha_{S}\left(m_{f}\right)$, and obtain interesting results at the light quark masses.

Our paper is organized as follows. In Section (II) we very briefly expose our approach to the analysis of vector meson production. In Section (III) we show that, with the appropriate reductions, our calculations lead to a description of these processes which exhibits a universality determined by properties of the overlap of photon and vector meson wave functions. Section (IV) concludes with a summary and discussion of our results. In the Appendix we present details of the vector meson light cone wave functions.

\section{BASIC FORMULÆ AND RESULTS}

For convenience we present here some basic formulae and results developed in our previous work on photo- and electroproduction [19, 20, 23, 24, 25, 26], where details 
can be found.

The amplitude for electroproduction of a vector meson $\mathrm{V}$ in polarization state $\lambda$ is written in our framework

$$
T_{\gamma^{*} p \rightarrow V p, \lambda}\left(s, t ; Q^{2}\right)=\int d^{2} \mathbf{R}_{1} d z_{1} \rho_{\gamma^{*} V, \lambda}\left(Q^{2} ; z_{1}, \mathbf{R}_{1}\right) J\left(s, \mathbf{q}, z_{1}, \mathbf{R}_{1}\right)
$$

with

$$
J\left(s, \mathbf{q}, z_{1}, \mathbf{R}_{1}\right)=\int d^{2} \mathbf{R}_{2} d^{2} \mathbf{b} e^{-i \mathbf{q} \cdot \mathbf{b}}\left|\psi_{p}\left(\mathbf{R}_{2}\right)\right|^{2} S\left(s, b, z_{1}, \mathbf{R}_{1}, z_{2}=1 / 2, \mathbf{R}_{2}\right) .
$$

Here $\psi_{p}\left(\mathbf{R}_{2}\right)$ is the proton wave function and

$$
\rho_{\gamma^{*} V, \lambda}\left(Q^{2} ; z_{1}, \mathbf{R}_{1}\right)=\psi_{V \lambda}\left(z_{1}, \mathbf{R}_{1}\right)^{*} \psi_{\gamma^{*} \lambda}\left(Q^{2} ; z_{1}, \mathbf{R}_{1}\right)
$$

represents the overlap of photon and vector meson wave functions; $S\left(s, b, z_{1}, \mathbf{R}_{1}, 1 / 2, \mathbf{R}_{2}\right)$ is the scattering amplitude of two dipoles with separation vectors $\mathbf{R}_{1}, \mathbf{R}_{2}$, colliding with impact parameter vector $\mathbf{b}[25$; $\mathbf{q}$ is the momentum transfer

$$
t=\left(p_{V}-p_{\gamma}^{*}\right)^{2}=-\mathbf{q}^{2}-m_{p}^{2}\left(Q^{2}+M_{V}^{2}\right) / s^{2}+O\left(s^{-3}\right) \approx-\mathbf{q}^{2}
$$

In the expressions above, $z$ is the longitudinal momentum fraction of the quark in the virtual photon and in the vector meson and $Q^{2}=-p_{\gamma}^{* 2}$ is the photon virtuality. The differential cross section is given by

$$
\frac{d \sigma}{d|t|}=\frac{1}{16 \pi s^{2}}|T|^{2}
$$

Here, $s$ is the center of mass energy of the $\gamma^{*}$-proton system.

The form of overlap written in Eq. 2.3) corresponds to SCHC (s-channel helicity conservation); generalizations can be made introducing a matrix in the polarization indices. In principle a three quark wave function should be used for the proton but earlier investigations have shown that a quark-diquark structure leads to fenomenologically consistent results and we adopt this model [25].

The light cone wave functions of the photon and vector meson have been previously discussed, and are presented in the Appendix to keep the paper self contained. Here we write only the scalar part of the meson wave function proposed by Brodsky and Lepage [27], which is used in the present calculations:

$$
\phi_{B L}(z, r)=\frac{N}{\sqrt{4 \pi}} \exp \left[-\frac{m_{f}^{2}\left(z-\frac{1}{2}\right)^{2}}{2 z \bar{z} \omega^{2}}\right] \exp \left[-2 z \bar{z} \omega^{2} r^{2}\right] .
$$


In previous papers [25, 26], another form of meson wave function has been used : the Bauer-Stech-Wirbel (BSW) [28, 29]. Since it results in no essential difference for the purposes here considered, we concentrate on the BL wave function.

In the expression above $m_{f}$ is the quark mass and $\bar{z}=1-z$. We note that $\phi_{B L}$ has two parameters: the normalization constant $N$ ( fixed by the wave function normalization) and the transverse size parameter $\omega$. In order to determine $\omega$ [23, 24, 25] we turn to the Van Royen-Weisskopf formula (including color) [30] which relates the leptonic decay width $\Gamma_{V \rightarrow e^{+} e^{-}}$to the wave function at the origin $\psi(0)$

$$
\Gamma\left(V \rightarrow e^{+} e^{-}\right)=\frac{16 \pi \alpha^{2} \hat{e}_{V}^{2}|\psi(0)|^{2}}{M_{V}^{2}}
$$

where $\alpha=1 / 137.036, \hat{e}_{V}$ is the effective charge in the vector meson, in units of the elementary charge $e$ and $M_{V}$ is the vector meson mass. This relation is the lowest order static approximation for the electromagnetic leptonic decay width of a vector meson [31].

This condition on the wave function, which determines $\omega$, is related to $f_{V}$, the coupling of the vector meson to the electromagnetic current, defined through

$$
<0\left|J_{e m}^{\mu}(0)\right| V(q, \lambda)>=e f_{V} M_{V} \epsilon^{\mu}(q, \lambda)
$$

If we use Eq. 2.8 in the $\mathrm{S}$ matrix element $<e^{+}(p, s) e^{-}(p /, s \prime)|S| V(q, \lambda)>$ and then calculate the decay rate of the vector meson, we obtain the relation of $f_{V}$ to $\Gamma_{V \rightarrow e^{+} e^{-}}[24$

$$
f_{V}^{2}=\frac{3 M_{V} \Gamma_{V \rightarrow e^{+} e^{-}}}{4 \pi \alpha^{2}}
$$

¿From Eqs. 2.9 and 2.7 we then obtain

$$
f_{V}^{2}=\frac{3 M_{V} \Gamma_{V \rightarrow e^{+} e^{-}}}{4 \pi \alpha^{2}}=\left(\frac{12}{M_{V}}\right) e_{V}^{2}|\psi(0)|^{2}
$$

relating $f_{V}, \Gamma_{V \rightarrow e^{+} e^{-}}$and $\psi(0)$.

Mesons in different states of polarization are represented by different wave functions. Consequentely, $N$ and $\omega$ are different for transverse and longitudinal states.

The relations between $f_{V}$ and the wave functions are [19, 25]

$$
f_{V}^{T}=\hat{e}_{V} \frac{N}{4 \pi} \frac{\sqrt{6}}{M_{V}} \int_{0}^{1} \frac{d z}{z \bar{z}}\left[2 \omega^{2}\left(z^{2}+\bar{z}^{2}\right)(4 z \bar{z})^{2}+m_{f}^{2}\right] \exp \left[-\frac{m_{f}^{2}\left(z-\frac{1}{2}\right)^{2}}{2 z \bar{z} \omega^{2}}\right]
$$

in the transverse case and

$$
f_{V}^{L}=\hat{e}_{V} \frac{N}{4 \pi} \sqrt{3} 16 \omega \int_{0}^{1} d z z \bar{z} \exp \left[-\frac{m_{f}^{2}\left(z-\frac{1}{2}\right)^{2}}{2 z \bar{z} \omega^{2}}\right]
$$


in the longitudinal case.

Numerically, we use $f_{V}^{T}=f_{V}^{L}=f_{V}$, with $f_{V}$ calculated through Eq. 2.9. The values thus obtained, together with other vector meson data, are presented in Table [I], in the Appendix. There, we also show in Table III our results for $N, \omega$, and the radius for each meson in each state, calculated through the wave function normalization equations, Eqs. 2.11 and (2.12) above (with $f_{V}$ from Table II and the mean value of the radius $r$. For the heavy quarks we take the renormalized masses $m_{c}$ $=1.25 \mathrm{GeV}, m_{b}=4.2 \mathrm{GeV}$ and for the light quarks we take the effective masses determined [32] as $m_{u}=m_{d}=0.2, m_{s}=0.3 \mathrm{GeV}$.

For the quark-diquark proton wave function, fixing the longitudinal momentum fraction at $z \approx \frac{1}{2}$ and making a Gaussian ansatz, we have

$$
\psi_{p}(r)=\frac{1}{2 \pi} \frac{1}{S_{p}} \exp \left[\frac{-r^{2}}{\left(2 S_{p}\right)^{2}}\right],
$$

where the size parameter is chosen as $S_{p}=0.74 \mathrm{fm}[33$.

In our model the energy dependence is motivated by the two-pomeron approach of Donnachie and Landshoff [34]. The hard pomeron leads to $\left(s / s_{0}\right)^{0.42}$ for $R_{1} \leq r_{c}$ and the soft pomeron leads to $\left(s / s_{0}\right)^{0.0808}$ for large dipoles, so that the integration over $R_{1}$ in Eq. (2.1) is split into two parts [25]. We use the numerical values $r_{c} \approx 0.22$ fm and $s_{0}=(20 \mathrm{GeV})^{2}$, taken from 35 .

\section{UNIVERSALITY}

As said above, we have used a nonperturbative QCD framework to calculate the observables of vector meson photo- and electroproduction, and we have obtained very good description of the data in all cases [19, 20, 23, 24, 25, 26]. Fig. 1] shows the data and the theoretical calculations of the electroproduction of $\rho, \omega, \phi, J / \psi$ and $\Upsilon$ vector mesons.

The quantitative predictions made in a unique way for different kinds of vector mesons cover 5 orders of magnitude in the cross sections [19]. The nonperturbative calculation leads to cross sections that plotted against $Q^{2}+M_{V}^{2}$ are nearly parallel straight lines, a result confirmed by experiments. Our work has shown that overlap of photon and vector meson wave functions alone determines large part of the behavior observed in these processes. This simple structure suggests a search for universality. 
FIG. 1: Integrated elastic cross sections in vector meson electroproduction: experimental data and theoretical calculations with the stochastic vector model.
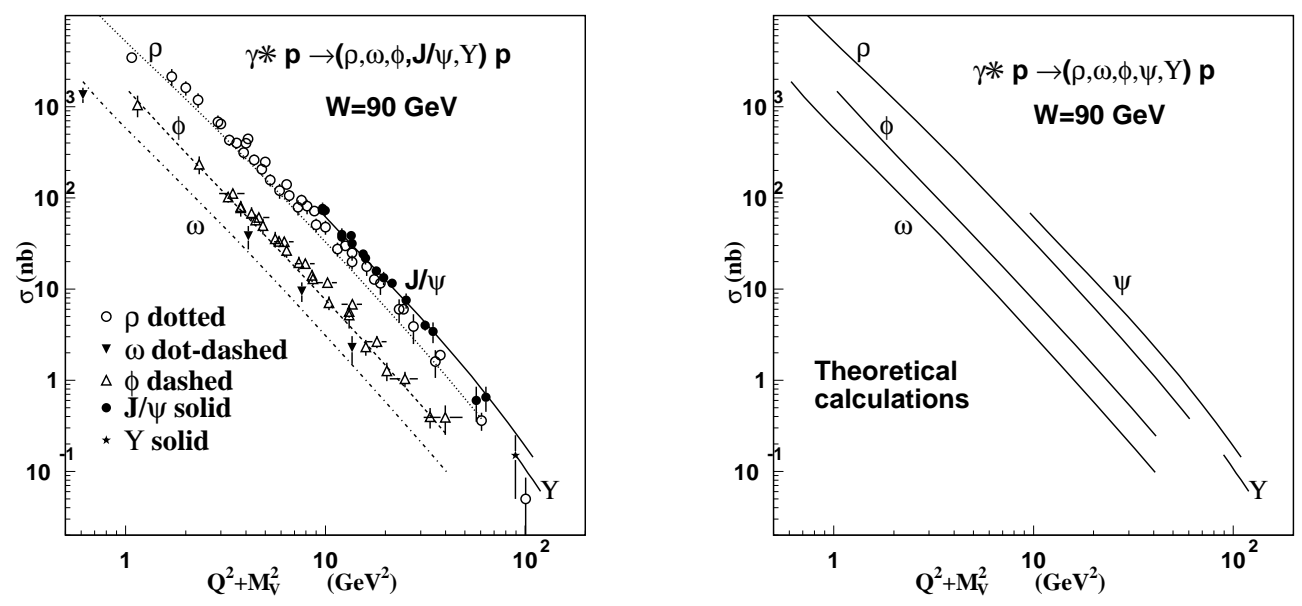

The effect of factorization in the magnitudes of the cross sections is exhibited with the extraction of charge factors $\hat{e}_{V}^{2}=1 / 2,1 / 18,1 / 9,4 / 9,1 / 9$ for $\rho, \omega, \phi, \psi$, and $\Upsilon$, respectively. Fig. 2 shows the theoretical results for all vector mesons after charge reduction, with approximately equal values for all cross sections. Since it can be seen from Fig. 1 that the experimental values agree very well with the theoretical predictions the universality to be derived from the theoretical expressions reflects an universality in the data. The agreement of our nonperturbative approach with the experimental results is a consequence also of the construction of the meson wave function, whose shapes are fixed by the experimental values of the electromagnetic leptonic decay rates of vector mesons $\Gamma_{V \rightarrow e^{+} e^{-}}$. These quantities fix the transverse size parameter $\omega$ contained in the wave function, as explained above [23, 24, 25]. The coupling of the vector meson to the electromagnetic current $\left(f_{V}\right)$ is related to $\Gamma_{V \rightarrow e^{+} e^{-}}$as shown in Eq. 2.10). The values obtained for $f_{V}$ are collected in Table II in the Appendix.

Fig. 3 shows the theoretical calculations of the electroproduction of $\rho, \omega, \phi, J / \psi$ and $\Upsilon$ vector mesons divided by the values of $f_{V}^{2}$. We observe a remarkable universality, closer than obtained with extraction of factors of squared charges. The small 
FIG. 2: Extraction of charge factors $e_{V}^{2}$ from the integrated elastic cross sections in vector meson electroproduction.

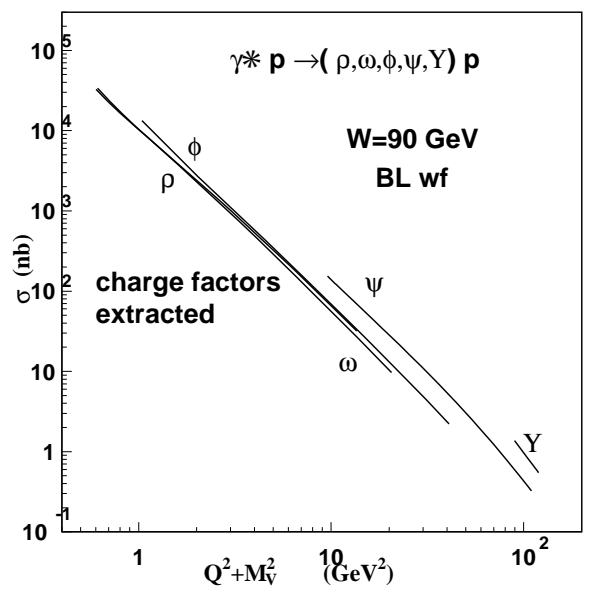

remaining splittings may be due to flavor dependent corrections in the calculations of the electromagnetic decay rates. We take these into account in the discussion that follows. The leading QCD radiative correction can be calculated in perturbation theory [21, 22, 36, 37] and causes a significant suppression in the value of $\Gamma$ given by the zero-order equation (2.7), which becomes

$$
\Gamma\left(V \rightarrow e^{+} e^{-}\right)=\Gamma_{0}\left[1-\left(\frac{16}{3 \pi}\right) \alpha_{S}\left(m_{f}\right)\right]
$$

leading to

$$
f_{V}^{2}=f_{V}^{(0) 2} F\left(m_{f}\right) \approx f_{V}^{(0) 2}\left[1-\left(\frac{16}{3 \pi}\right) \alpha_{S}\left(m_{f}\right)\right]
$$

The flavor dependence comes through the running strong coupling constant $\alpha_{S}$, which depends on $m_{f}$. Since $\alpha_{S}$ is known at the mass values of the $b$ and $c$ quark masses 38]

$$
\alpha_{S}\left(m_{b}\right)=0.23 \pm 0.01 \quad, \quad \alpha_{S}\left(m_{c}\right)=0.33 \pm 0.01
$$

the corrected Eq. (3.2) can be applied directly to $\Upsilon$ and $J / \psi$.

Fig. 4 shows the theoretical calculations of electroproduction of $J / \psi$ and $\Upsilon$ vector mesons with extraction of $f_{V}^{(0) 2}$ factors, with remarkable coincidence of the reduced 
FIG. 3: Extraction of $f_{V}^{2}$ coupling factors from the integrated elastic cross sections in vector meson electroproduction.

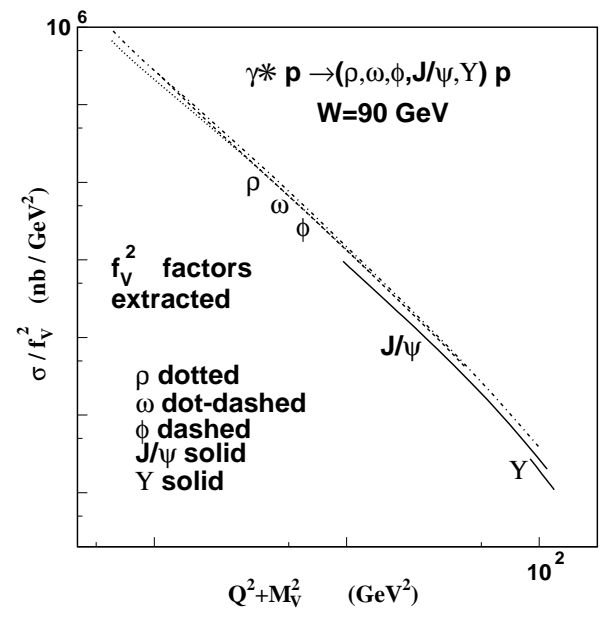

values of the cross sections. We thus have precise universality for $\Upsilon$ and $J / \psi$ mesons. The simple behavior connecting $J / \psi$ and $\Upsilon$ cross sections, that we explained by sheer acting on wave function construction, is a manifestation of the factorization that shows that the whole $Q^{2}$ dependence of the cross sections is determined by the overlap of the photon and vector meson wave functions. In Fig. 5 we exhibit the accuracy of the calculation of the $J / \psi$ cross section using only the overlap strength of photon and $J / \psi$ wave functions (the overlap strength is formed by the integration over the internal variables of the $q \bar{q}$ pairs of the overlap function multiplied by $r^{2}$ ) [20]. The accurate calculation of the cross section with the stochastic vacuum model in the LHS is compared to the calculation with the overlap strengths in the RHS. A numerical factor 24000 takes into account the interaction of the proton with the QCD field, which has no $Q^{2}$ dependence. Actually the $Q^{2}$ dependence of cross sections is of the form

$$
\sigma=\frac{A_{V}}{\left(Q^{2}+M_{V}^{2}\right)^{n}}
$$

which is contained in the shape of the integration of products of the modified Bessel functions $K_{0}$ and $K_{1}$ with powers and Gaussians that appear in the overlap strengths. 
FIG. 4: Extraction of the bare $f_{V}^{(0) 2}$ coupling factors from the integrated elastic cross sections of $J / \psi$ and $\Upsilon$ mesons .

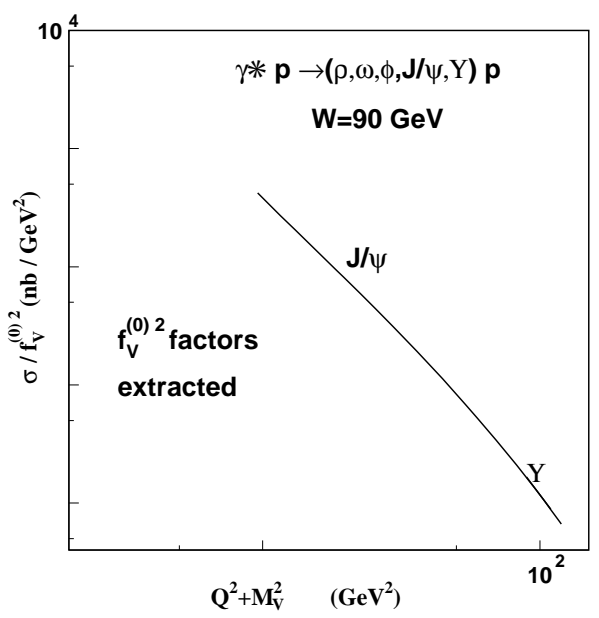

FIG. 5: Data and calculation of $J / \psi$ cross section as a function of photon virtuality . In the RHS we show the $Q^{2}$ dependence of the overlap strength determined only by wave functions.
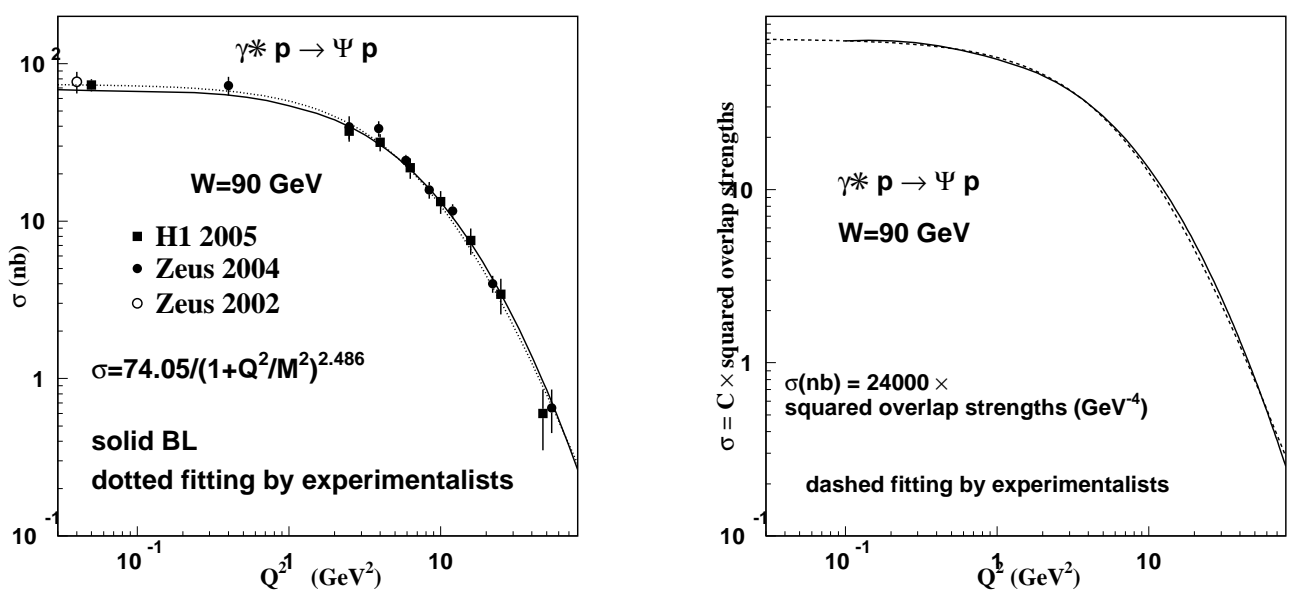
Good results for cross sections calculated only with these quantities are obtained whenever the range of the overlap region is small compared to the typical range of the nonperturbative interaction governing the process [20].

Let us next consider the light vector mesons. Here we may have some complications, since it may be necessary to include relativistic and higher-order radiative corrections to $f_{V}$. Moreover, we will need the values of $\alpha_{S}$ for small $m_{f}$, which are not yet determined experimentally.

While there is a general agreement as to the high energy behavior of the strong coupling constant [38, 39, 40], the infrared region, where $\alpha_{S}\left(Q^{2}\right)$ develops singularities, is not determined. Perturbative calculations point to a singular behaviour, but the possibility of finite values at small $Q^{2}$ has been extensively studied in recent years. There are analytical, phenomenological and lattice calculations exploring this possibility, and many arguments and techniques have been proposed to solve the problem of the elimination of the infrared singularities [39, 41].

A phenomenological proposal to determine the infrared strong coupling constant is that of the so called physical coupling or effective charge, which consists in defining $\alpha_{S, \text { eff }}\left(Q^{2}\right)$ from a given physical observable [42]. This was analysed in connection to the renormalization-scheme dependence problem in perturbative field theories [43, 44]. This effective charge is a resummation in perturbation theory [45, 46, 47]. Brodsky et al. argue that a perturbatively calculable physical quantity can be used to define an effective charge by incorporating the entire radiative correction in its definition [45].

In line with this phenomenological proposal, for the light vector mesons we define an effective strong running coupling constant that absorbs the higher order corrections to $f_{V}$ and so, instead of the corrected Eq. (3.1), we write

$$
\Gamma\left(V \rightarrow e^{+} e^{-}\right)=\Gamma_{0}\left[1-\left(\frac{16}{3 \pi}\right) \alpha_{S, \text { eff }}\left(m_{f}\right)\right]
$$

Now the reduction factor in Eq. 3.2 is written as

$$
F\left(m_{f}\right) \approx\left[1-\left(\frac{16}{3 \pi}\right) \alpha_{S, \mathrm{eff}}\left(m_{f}\right)\right]
$$

and is determined by fitting that leads to universality of cross sections. The results obtained are shown in Table III. With this choice for the factors that account for the radiative corrections to the electromagnetic decays of the light vector mesons, 
TABLE I: Reduction factors of $\Gamma$ for $\phi, \rho$ and $\omega$

\begin{tabular}{|ccc|}
\hline Meson $m_{f}(\mathrm{GeV})$ & $F$ (fitted) \\
\hline$\phi$ & 0.3 & $0.32 \pm 0.01$ \\
$\rho$ & 0.2 & $0.32 \pm 0.01$ \\
$\omega$ & 0.2 & $0.29 \pm 0.01$ \\
\hline
\end{tabular}

FIG. 6: Extraction of renormalized $f_{V}^{(0) 2}$ coupling factors from the integrated elastic cross sections of electroproduction of all vector mesons: theoretical results and experimental data converted with same factors for each vector meson, as described in the text.
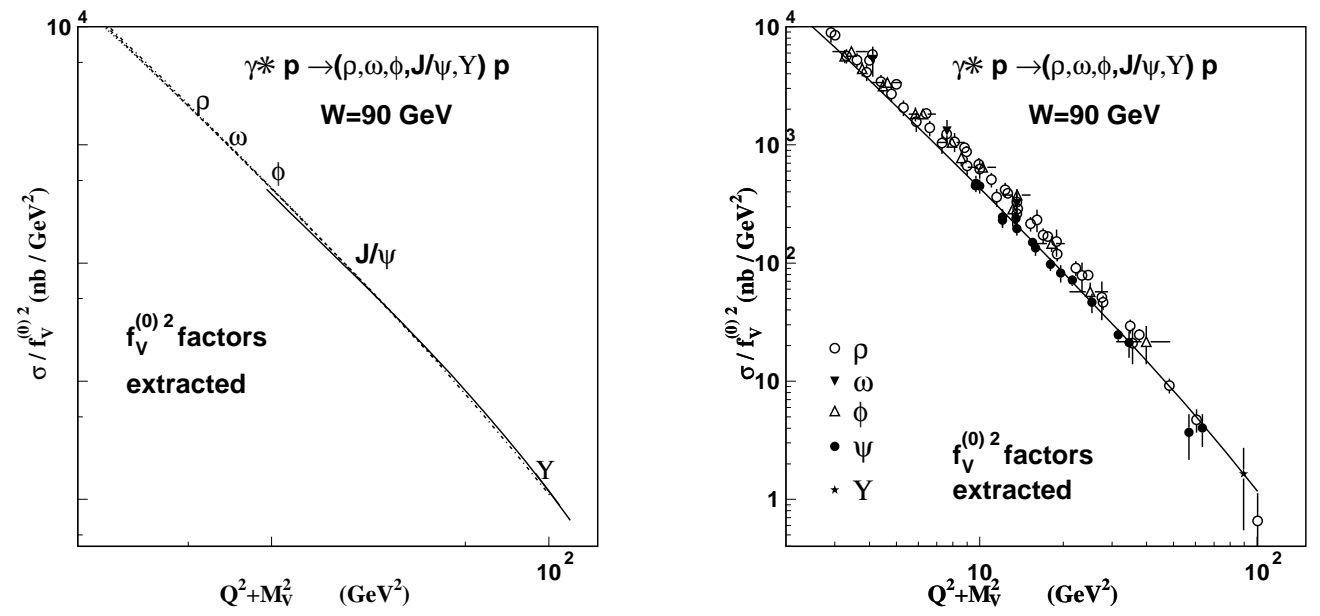

we have the renormalized couplings $f_{V}^{(0) 2}$ for all mesons and then we may put the electroproduction cross section of all five vector mesons in a single line, as shown in Fig. 6. Through the fitted values of $F\left(m_{f}\right)$ we obtain values of the effective strong coupling constant at low masses

$$
\alpha_{S, \text { eff }}\left(m_{f}\right) \approx 0.4 \quad, \quad \text { for } m_{f} \approx(0.2-0.3) \mathrm{GeV} \text {. }
$$

This result is similar to values found in the literature.

On the theoretical side, there are many calculations leading to a region below $\alpha_{S}\left(Q^{2}\right) \approx 1$ for low values of $Q^{2}$. Let us explicitly mention a few of these. 
In a classical paper [48, studying gauge invariant Schwinger-Dyson equations, Cornwall advocated the dynamical generation of an effective gluon mass, resulting in a gluon propagator that reaches a finite, non vanishing value in the deep infrared and in $\alpha_{S}\left(Q^{2}\right)$ which is asymptotically free in the ultraviolet and attains a finite infrared value. This general picture is confirmed by using large volume lattices, for $\mathrm{SU}(2)$ [49] and $\mathrm{SU}(3)$ [50] pure Yang-Mills. Other recent studies are in agreement with Cornwall results [41, 51, 52, 53].

According to the original Cornwall proposal, we have

$$
\frac{\alpha_{S}\left(M_{Z_{0}}\right)}{\alpha_{S}\left(Q^{2}\right)}=1+\frac{\alpha_{S}\left(M_{Z_{0}}\right) \beta_{0}}{2 \pi} \ln \frac{Q^{2}+4 m_{g}^{2}}{M_{Z_{0}}} .
$$

Here, $M_{Z_{0}}$ is the mass of $Z_{0}$ used as reference, $\beta_{0}=11-\frac{2}{3} n_{f}$, with $n_{f}=$ number of flavors, and $m_{g}$ is the gluon mass, which can be neglected in face of $M_{Z_{0}}$.

This infrared finite strong coupling constant has been used to study the asymptotic pion form factor [54], resulting in a better agreement with the data.

The values of the strong coupling constants that appear in the radiative corrections to the electromagnetic decays, which modify the effective couplings, are exhibited in Fig. 7. In the figure we compare the values here obtained with measurements and plot the perturbative and nonperturbative [48] calculations. In the nonperturbative case we use $m_{g}=0.6 \mathrm{GeV}$. The figure shows that the value of $\alpha_{S \text {,eff }} \approx 0.4$ for low masses, obtained by our fitting, is in the range of values predicted by Cornwall [48] and by more recent work. In a later paper [55], and using a different technique (the functional Schrödinger equation), Cornwall estimated $\alpha_{S}\left(M^{2}\right) \approx 0.4$ for $\mathrm{M}=$ $0.6 \mathrm{GeV}$, which is again in agreement with our result. The same is true for ref. [52], where $\alpha_{S}$ is found to be a bit below 0.4 for $Q^{2} \approx 0.1 \mathrm{GeV}^{2}$. In a recent paper [53], calculations of QCD effective charges performed within two different theoretical frameworks are shown to acquire a common value $\alpha_{S}(0)=0.7 \pm 0.3$.

On the phenomenological side, several different analyses of scattering data sensitive to low momentum effects point to values of $\alpha_{S}\left(Q^{2}=0\right)$ in the range [41].

$$
\alpha_{S}(0) \approx 0.7 \pm 0.3
$$

which is in agreement with our values.

In addition, we mention that the underlying stochastic vacuum model also predicts finite strong coupling $\alpha_{S} \approx 0.8$ in the infrared region [56]. 
FIG. 7: Strong running coupling constant. The dashed lines are the usual perturbative expresions, and the solid line represents Eq. 3.5 with $m_{g}=0.6 \mathrm{GeV}$.

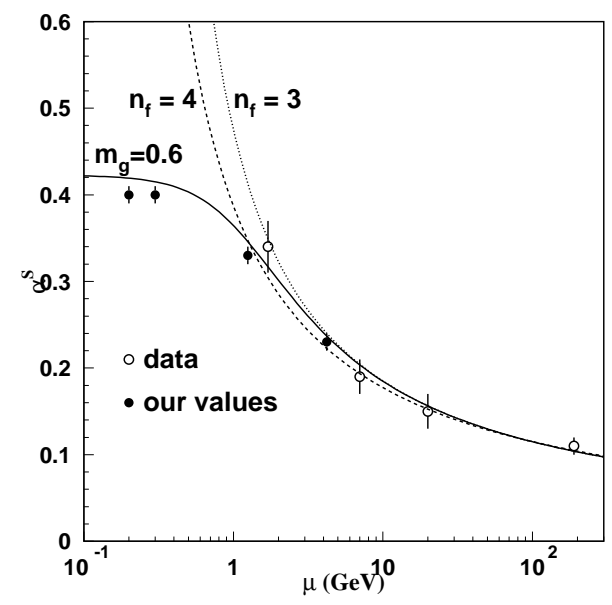

\section{CONCLUSIONS}

Our treatment of elastic vector meson electroproduction stresses nonperturbative aspects of the QCD calculation, showing that large part of the description of this process is supplied by the hadronic structure, namely, the wave functions of photons and vector mesons and a simple diquark model for the proton. Details of the structure of the proton, that participates in the processes like an overall object, determined by its size only, do not enter in the description of the integrated observables.

A full description of the framework of the calculations can be found in previous papers [6, 20, 23, 25, 26]. The calculations, without free parameters, show that quite simple features are able to explain the data, covering a wide range of 5 orders of magnitude in the cross sections[19].

Each of the different mesons enter in the calculation characterized only by the masses and charges of its quark contents, and with their normalized wave function individualized only by the corresponding electromagnetic decay rate (related to the value of the wave function at the origin). The main features of the $Q^{2}$ dependence of 
electroproduction of vector mesons are contained in the overlap integral of the light cone wave functions of photons and mesons folded with the basic $r^{2}$ behaviour of the dipole cross sections [20].

In this paper we concentrate on the integrated elastic cross sections, studying its universality in the $Q^{2}$ dependence. The energy dependence, which is well described by the two-pomeron model, is not detailed here : we choose a value $\mathrm{W}=90 \mathrm{GeV}$ for which experiments are available for all vector mesons.

Our calculation gives a unified description of the electroproduction for all five S-wave vector mesons. The $Q^{2}$ dependence of the integrated elastic cross section shows a regular shape when plotted against the variable $Q^{2}+M_{V}^{2}$. The lines are almost parallel, and it is well known that, after extraction of factors equal to the squared electric charges of the constituent quarks in the vector mesons (with appropriate averages in the $\rho$ and $\omega$ cases), the reduced cross sections tend to concentrate near a common line. We show that this approximate universality becomes more exact when the extracted factor is the square of the electromagnetic coupling $f_{V}$. This improved universality is interesting because this factor is more meaningful than the squared charge, since it is connected to the value of the wave function at the origin, entering in the determination of the basic parameter $\omega$ of the wave function, and fixing the size of the vector meson.

The numerical value of the coupling $f_{V}$ for each vector meson is fixed by the experimental value of the electromagnetic decay rate $\Gamma_{V \rightarrow e^{+} e^{-}}$. This experimental value is affected by radiative corrections at the electromagnetic vertex, which depend on the masses of the intervening quarks. We remark that a more exact universality would be observed if these corrections were not present.

The criterion of universality of reduced cross sections imposed for the light meson cases, using the same expression for the radiative correction, leads to effective values for the strong coupling constant at the low mass values. These values are in iteresting agreement with theoretical studies concerning the infrared behavior of the gluon propagator.

The successful description of electroprodution cross sections, without free parameters, including all vector mesons, and the universality property that is inherent to our nonperturbative framework, shows that microscopic details are not important for the observables here studied. Only the dipole distribution in the wave functions 
and the hadronic sizes, and the range of the interaction mediated by the nonperturbative QCD vacuum field, are relevant for the phenomenology.

\section{Acknowledgments}

The authors wish to thank CNPq (Brazil) and FAPERJ (Brazil) for support of the scientific collaboration program between Heidelberg and Rio de Janeiro groups working on hadronic physics. One of the authors (EF) is grateful to CNPq (Brazil) for research fellowship and grant.

Photon and vector meson light-cone wave functions

\section{APPENDIX A: PHOTON AND VECTOR MESON LIGHT-CONE WAVE FUNCTIONS}

The color part of the photon wave function can be treated separately and the result is an overall multiplicative factor $\sqrt{N_{c}}$. The helicity and spatial configuration part of the wave function $\psi_{\gamma^{*}, \lambda}\left(Q^{2} ; z, r, \theta\right)$ is calculated in light-cone perturbation theory. In lowest order, for each polarization $\lambda$, we write [13, 23, 27, [57, 58, 59],

$$
\begin{aligned}
\psi_{\gamma^{*}, 1}\left(Q^{2} ; z, r, \theta\right)= & \hat{e}_{f} \frac{\sqrt{6 \alpha}}{2 \pi}\left[i \epsilon e^{i \theta}\left(z \delta_{h,+} \delta_{\bar{h},-}-\bar{z} \delta_{h,-} \delta_{\bar{h},+}\right) K_{1}(\epsilon r)\right. \\
& \left.+m_{f} \delta_{h,+} \delta_{\bar{h},+} K_{0}(\epsilon r)\right], \\
\psi_{\gamma^{*},-1}\left(Q^{2} ; z, r, \theta\right)= & \hat{e}_{f} \frac{\sqrt{6 \alpha}}{2 \pi}\left[i \epsilon e^{-i \theta}\left(\bar{z} \delta_{h,+} \delta_{\bar{h},-}-z \delta_{h,-} \delta_{\bar{h},+}\right) K_{1}(\epsilon r)\right. \\
& \left.+m_{f} \delta_{h,-} \delta_{\bar{h},-} K_{0}(\epsilon r)\right]
\end{aligned}
$$

and

$$
\psi_{\gamma^{*}, 0}\left(Q^{2} ; z, r\right)=\hat{e}_{f} \frac{\sqrt{3 \alpha}}{2 \pi}(-2 z \bar{z}) \delta_{h,-\bar{h}} Q K_{0}(\epsilon r)
$$

where

$$
\epsilon=\sqrt{z \bar{z} Q^{2}+m_{f}^{2}}
$$

$\alpha=1 / 137.036$ and $m_{f}$ is the quark mass, $\hat{e}_{f}$ is the quark charge in units of the elementary charge for each flavor $f ; z(\bar{z})$ is the quark (antiquark) longitudinal momentum fraction; $h(\bar{h})$ is the quark (antiquark) helicity; $K_{0}$ and $K_{1}$ are the modified Bessel functions. In the longitudinal case there is an additional $\delta^{2}(\vec{r})$ which ca be 
dropped because the color interaction vanishes at zero transverse distance. The quark masses we use are $m_{u}=m_{d}=0.2, m_{s}=0.3, m_{c}=1.25$ and $m_{b}=4.2$ (values in $\mathrm{GeV})$.

Meson wave functions are more model dependent than photon wave functions. We take for the vector meson the spin structure from the vector current leading to expressions similar to those of of the photon [23, 57? ].

$$
\begin{aligned}
\psi_{V,+1}(z, r, \theta)= & \left(-i e^{i \theta} \partial_{r}\left(z \delta_{h,+} \delta_{\bar{h},-}-\bar{z} \delta_{h,-} \delta_{\bar{h},+}\right)\right. \\
& \left.+m_{f} \delta_{h,+} \delta_{\bar{h},+}\right) \phi_{V}(z, r) \\
\psi_{V,-1}(z, r, \theta)= & \left(-i e^{-i \theta} \partial_{r}\left(\bar{z} \delta_{h,+} \delta_{\bar{h},-}-z \delta_{h,-} \delta_{\bar{h},+}\right)\right. \\
& \left.+m_{f} \delta_{h,-} \delta_{\bar{h},-}\right) \phi_{V}(z, r)
\end{aligned}
$$

and

$$
\psi_{V, 0}(z, r)=\left(\omega 4 z \bar{z} \delta_{h,-\bar{h}}\right) \phi_{V}(z, r)
$$

In this paper we use the functional form for the scalar function $\phi_{V}(z, r)$ proposed by Brodsky and Lepage [27]

$$
\phi_{B L}(z, r)=\frac{N}{\sqrt{4 \pi}} \exp \left[-\frac{m_{f}^{2}\left(z-\frac{1}{2}\right)^{2}}{2 z \bar{z} \omega^{2}}\right] \exp \left[-2 z \bar{z} \omega^{2} r^{2}\right]
$$

It contains two parameters, $N$ and $\omega$, which are determined, as explained in section 2 , by the normalization condition and the leptonic decay width, $\Gamma_{V \rightarrow e^{+} e^{-}}$, which is related to $f_{V}$, the coupling of the vector meson to the electromagnetic current. Table II] shows vector meson data and the values obtained for $f_{V}$ through eq. 2.9. Numerically,we use $f_{V}^{T}=f_{V}^{L}=f_{V}$, with $f_{V}$ calculated through eq. 2.9. The values thus obtained, together with other vector meson data, are presented in Table [II. Table III shows our results for $\omega, N$, and radius $r$ for each meson in each state, calculated with eqs. 2.11 and 2.12, the wave function normalization equation $\int_{0}^{1} d z \int d^{2} \mathbf{r}\left|\psi_{V, \lambda}(z, \mathbf{r})\right|^{2}=1$ and the mean value of $r$.

After summation over the helicity indices, the overlaps of the photon and vector meson wave functions (eq. 2.3) are

$$
\rho_{\gamma^{*} V, \pm 1}\left(Q^{2} ; z, r\right)=\hat{e}_{V} \frac{\sqrt{6 \alpha}}{2 \pi}\left(4 \epsilon_{f} \omega^{2} r z \bar{z}\left[z^{2}+\bar{z}^{2}\right] K_{1}\left(\epsilon_{f} r\right)+m_{f}^{2} K_{0}\left(\epsilon_{f} r\right)\right) \phi_{B L}(z, r(\mathcal{A} \mathrm{A} 8)
$$


TABLE II: S-wave vector meson data. The coupling $f_{V}$ and the electromagnetic decay width $\Gamma_{V \rightarrow e^{+} e^{-}}$are related through eq.2.9. The quantity $\hat{e}_{V}$ is the effective quark charge in units of the elementary charge, determined by the $q \bar{q}$ structure of each meson.

\begin{tabular}{|lcccc|}
\hline Meson & $M_{V}(\mathrm{MeV})$ & $\hat{e}_{V}$ & $\Gamma_{V \rightarrow e^{+} e^{-}}(\mathrm{keV})$ & $f_{V}(\mathrm{GeV})$ \\
\hline$\rho(770)$ & $775.49 \pm 0.34$ & $1 / \sqrt{2}$ & $7.04 \pm 0.06$ & $0.15645 \pm 0.0043$ \\
$\omega(782)$ & $782.65 \pm 0.12$ & $1 / 3 \sqrt{2}$ & $0.60 \pm 0.02$ & $0.04588 \pm 0.0008$ \\
$\phi(1020)$ & $1019.455 \pm 0.020$ & $-1 / 3$ & $1.27 \pm 0.04$ & $0.07619 \pm 0.0012$ \\
$J / \psi(1 S)$ & $3096.916 \pm 0.011$ & $2 / 3$ & $5.55 \pm 0.141$ & $0.27759 \pm 0.0035$ \\
$\Upsilon(1 S)$ & $9460.30 \pm 0.26$ & $-1 / 3$ & $1.340 \pm 0.018$ & $0.23839 \pm 0.0016$ \\
\hline
\end{tabular}

TABLE III: Parameters of the vector meson wave functions

\begin{tabular}{|lccccccc}
\hline & \multicolumn{3}{c}{ Transverse } & \multicolumn{3}{c}{ Longitudinal } \\
& $\omega(\mathrm{GeV})$ & $N$ & radius $(\mathrm{fm})$ & $\omega(\mathrm{GeV})$ & $N$ & radius(fm) \\
\hline$\rho(770)$ & 0.2809 & 2.0820 & 1.0637 & 0.3500 & 1.8366 & 0.6402 \\
$\omega(782)$ & 0.2618 & 2.0470 & 1.1110 & 0.3088 & 1.8605 & 0.7194 \\
$\phi(1020)$ & 0.3119 & 1.9201 & 0.8569 & 0.3654 & 1.9191 & 0.5973 \\
$J / \psi(1 S)$ & 0.6452 & 1.4752 & 0.3418 & 0.7140 & 2.2769 & 0.2898 \\
$\Upsilon(1 S)$ & 1.3333 & 1.1816 & 0.1551 & 1.3851 & 2.7694 & 0.1456 \\
\hline
\end{tabular}

for the transverse case,

$$
\rho_{\gamma^{*} V, 0}\left(Q^{2} ; z, r\right)=-16 \hat{e}_{V} \frac{\sqrt{3 \alpha}}{2 \pi} \omega z^{2} \bar{z}^{2} Q K_{0}\left(\epsilon_{f} r\right) \phi_{B L}(z, r)
$$

for the longitudinal case.

The overlap strengths, formed by integration over the internal variables of the quarkantiquark pairs of the overlap function multiplied by $r^{2}$ are

$$
Y_{\gamma^{*} V, T}\left(Q^{2}\right)=\int_{0}^{1} d z \int d^{2} \mathbf{r} r^{2} \rho_{\gamma^{*} V, \pm 1}\left(Q^{2} ; z, r\right) \equiv \hat{e}_{V} \widehat{Y}_{\gamma^{*} V, T}\left(Q^{2}\right)
$$

for transverse polarization and

$$
Y_{\gamma^{*} V, L}\left(Q^{2}\right)=\int_{0}^{1} d z \int d^{2} \mathbf{r} r^{2} \rho_{\gamma^{*} V, 0}\left(Q^{2} ; z, r\right) \equiv \hat{e}_{V} \widehat{Y}_{\gamma^{*} V, L}\left(Q^{2}\right)
$$


for longitudinal polarization.

[1] O. Nachtmann, Annals of Physics 209 (1991) 436.

[2] C. Ewerz and O. Nachtmann, XI International Conference on Elastic and Diffractive Scattering, Blois, 2005 hep-ph/0511051.

[3] C. Ewerz and O. Nachtmann, Annals of Physics 322 (2007) 1635.

[4] H.G. Dosch, Phys. Lett. B 190 (1987) 177.

[5] H.G. Dosch and Y.A. Simonov, Phys. Lett. B 205 (1988) 339.

[6] H.G. Dosch, E. Ferreira and A. Krämer, Phys. Rev. D 50 (1994) 1992.

[7] H.G. Dosch, S. Donnachie, P. Landshoff and O. Nachtmann, Pomeron Physics and QCD, Cambridge University Press, Cambridge, England (2002).

[8] A.Di Giacomo, H.G. Dosch, V.I. Shevchenko and Y.A. Simonov, Phys. Rep.372 (2002) 319.

[9] A. Donnachie and H.G. Dosch, Phys. Lett. B 502 (2001) 74.

[10] A. Donnachie and H.G. Dosch, Phys. Rev. D 65 (2002) 014019.

[11] A. Donnachie and P. Landshoff, Phys. Lett. B 311 (1987) 403.

[12] M.G. Ryskin, Z.Phys. C 57 (1993) 89.

[13] S.J. Brodsky, L. Frankfurt, J.F. Gunion, A.H. Mueller and M. Strikman, Phys. Rev. D 50 (1994) 3134.

[14] M.G. Ryskin, R.G. Roberts, A.D. Martin and E.M. Levin, Z.Phys. C 76 (1997) 231.

[15] L. Frankfurt, A. Radyushkin and M. Strikman, Phys. Rev. D 55 (1997) 98.

[16] L. Frankfurt, W. Koepf and M. Strikman, Phys. Rev. D 57 (1998) 512.

[17] E. Gotsman, E. Ferreira, E. Levin, U. Maor and E. Naftali, Phys. Lett. B 503 (2001) 277.

[18] I.P. Ivanov, N.N. Nikolaev and A.A. Savin, Phys. Part. Nucl. 37 (2006) 1.

[19] H.G. Dosch and E. Ferreira, Eur. Phys. J. C 51 (2007) 83.

[20] E. Ferreira and V.L. Baltar, Nucl. Phys. A 748 (2005) 608.

[21] R. Barbieri et al., Phys. Lett. B 57 (1975) 455 ; Nucl.Phys. B 105 (1975) 125.

[22] W. Celmaster, Phys. Rev. D 19 (1979) 1517.

[23] H.G. Dosch, T. Gousset, G. Kulzinger and J.J. Pirner, Phys. Rev. D 55 (1997) 2602.

[24] G. Kulzinger, H.G. Dosch and H.J. Pirner, Eur. Phys. J. C 7 (1999) 73. 
[25] H.G. Dosch and E. Ferreira, Eur. Phys. J. C 29 (2003) 45.

[26] H.G. Dosch and E. Ferreira, Phys. Lett. B 576 (2003) 83.

[27] G.P. Lepage and S.J. Brodsky, Phys. Rev. D22 (1980) 2157.

[28] M. Wirbel, B. Stech and M. Bauer, Z. Phys. C29 (1985) 637.

[29] M. Bauer, B. Stech and M. Wirbel, Z. Phys. C34 (1987) 103.

[30] R.Van Royen and V.F. Weisskopf, Nuovo Cimento 50 (1967) 617.

[31] J.D. Jackson, SLAC Report 198, Proceedings of Summer Institute on Particle Physics (1976) 147.

[32] H.G. Dosch, T. Gousset and H.J. Pirner, Phys. Rev. D 57 (1998) 1666.

[33] H.G. Dosch, O. Nachtmann, T. Paulus and S. Weinstock, Eur. Phys. J. C 21 (2001) 339.

[34] A. Donnachie and P.V. Landshoff, Phys. Lett. B 437 (1998) 408.

[35] A. Donnachie and H.G. Dosh, Phys. Rev. D 65 (2002) 014019.

[36] W. Buchmüller and S.H.H. Tye, Phys. Rev. D 24 (1981) 132.

[37] F. Bissey, J.J. Dugne and J.F. Mathiot, Eur. Phys. J. C 24 (2002) 101.

[38] I. Hinchliffe and A. Manohar, Particle Data Group, Journal of Physics G,33 (2006) 110.

[39] G.M. Prosperi, M. Raciti and C. Simolo, Prog. Part. Nucl. Phys. 58 (2007) 387.

[40] S. Bethke, Prog.Part.Nucl.Phys. 58 (2007) 351.

[41] A.C. Aguilar, A. Mihara and A.A. Natale, Phys. Rev. D 65 (2002) 054011.

[42] G. Grumberg, Phys.Letts. B 95 (1980) 70.

[43] S.J. Brodsky, G.P. Lepage, P.B. Mackenzie and H.J. Lu, Phys. Rev. D 28 (1983) 228.

[44] G. Grumberg, Phys. Rev. D 29 (1984) 2315.

[45] S.J. Brodsky and H.J. Lu, Phys. Rev. D 51 (1995) 3652.

[46] S.J. Brodsky, C-R Ji, A. Pang and D. Robertson, Phys. Rev. D 57 (1998) 245.

[47] S.J. Brodsky, S. Menke, C. Merino and J. Rathsman Phys. Rev. D 67 (2003) 055008.

[48] J.M. Cornwall Phys. Rev. D 26 (1982) 1453.

[49] A. Cucchieri and T. Mendes, Proc.Sci.LATTICE2007 (2007) 297; Phys. Rev. Lett. 100 (2008) 241601; P.O. Bowman et al., Phys. Rev. D 76 (2007) 094505.

[50] I.L. Bogolubsky, E.M. Ingelfritz, M. Muller-Preussker and A. Sternbeck, Proc. Sci. LATTICE2007 (2007) 290; Phys. Lett. B 676 (2009) 69.

[51] A.C. Aguilar and J. Papavassiliou, J.HEP.12 (2006) 12.

[52] A.C. Aguilar, D. Binosi and J. Papavassiliou, Proc.Sci.LIGHT CONE 2008 (2008) 
50.

[53] A.C. Aguilar, D. Binosi, J. Papavassiliou and J. Rodriguez-Quintero , arXiv:hep$\mathrm{ph} / 09062633$.

[54] C. Ji and F. Amiri, in photon-photon collisions using a fozen coupling constant" Phys. Rev. D 42, 3764 (1990).

[55] J.M. Cornwall Phys. Rev. D 76 (2007) 025012.

[56] A.I. Shoshi, F.D. Steffen, H.G. Dosch and H.J. Pirner, Phys. Rev. D 68 (2003) 074004.

[57] N. N. Nikolaev and B. G. Zakharov, Z. Phys. C49 (1991) 607.

[58] J.D. Bjorken, J.B. Kogut and D.E. Soper, Phys. Rev. D3 (1971) 1382.

[59] G.P. Lepage et al., in Particles and Fields 2, Proceedings of the Banff Summer Institute, Banff, Canada, 1981, A.Z. Capri, A.N. Kamal (Eds), Plenum, New York, 1983. 